\title{
First Evaluation of the PTN-104 Plethysmographic Sensor for Heart Rate Measurement
}

\author{
Wiesław Szaj ${ }^{1}$, Wiktoria Wojnarowska ${ }^{2}$, Bogdan Pajdo ${ }^{1}$ \\ ${ }^{I}$ Department of Physics and Medical Engineering, Faculty of Mathematics and Applied Physics, Rzeszow University of \\ Technology,Powstancow Warszawy 8, 35-959 Rzeszow, Poland,wszaj@prz.edu.pl \\ ${ }^{2}$ Doctoral School of Engineering and Technical Sciences at the Rzeszow University of Technology, Powstancow Warszawy \\ 8,35-959 Rzeszow,Poland,d510@stud.prz.edu.pl
}

\begin{abstract}
The purpose of this study was to examine the accuracy of HR measurements by the PTN-104 sensor in comparison to the fingertip pulse oximeter, which is a photoplethysmographic sensor (PPG). Twelve healthy participants underwent the same protocol during a single visit. Measurements were taken after each participant completed an initial rest period of 5 minutes and after 1-minute of exercising comprising of 30 squats. Each subject had the PTN-104 sensor attached to the index finger and a fingertip pulse oximeter to the opposite one. When examining the data in aggregate, there was a strong correlation between the PTN-104 sensor and PPG for HR $(r=0.988)$ with a mean bias of $-2.55 \mathrm{bpm}(95 \% \mathrm{LoA}+5.0,-10.1)$. The PTN-104 sensor satisfied validity criteria for HR monitors, however, showed a lower accuracy for measurements at rest, which is surprising. Due to the noticed limitations, this study should be repeated with a larger group of subjects and the PTN-104 sensor should be compared to the gold standard method for measuring HR, which is ECG.
\end{abstract}

Keywords: Data acquisition, vital signal, photoplethysmography (PPG), pulse oximeter, measurement validation, physiological measurement.

\section{INTRODUCTION}

Heart rate (HR) is defined as the frequency of heartbeats over a specific time interval, usually expressed in beats per minute. It is the wave of blood in the artery created by the contraction of the left ventricle during the cardiac cycle. This vital sign is a determinant of myocardial oxygen demand, coronary blood flow, myocardial efficiency, and is crucial for adjusting cardiac output to metabolic demands [1].

HR is regulated by the autonomic nervous system and can therefore be easily influenced by various disease conditions [1]. It is an important parameter in the assessment of heart health. There is evidence that Resting Heart Rate (RHR) can be used for the prediction and diagnostics of some disease conditions [2], [3]. A healthy heart makes a specific pattern of waves on the recording, a damaged or diseased heart changes that pattern in recognizable ways. In patients with heart failure (HF), increased RHR is a significant predictor of all-cause mortality in ambulatory patients on optimal medical therapy [4], whereas, in the general population, high RHR is associated with an increased risk of death and cardiovascular events in men [5]. Moreover, RHR is a potentially useful measure of neurological performance [6]. Besides, measurements of HR are used in a test of the level of physical fitness.
RHR varies from person to person. The normal RHR is considered in the range of $70-100$ beats per minute (bpm) in children and $60-100 \mathrm{bpm}$ in adults (over 18 years of age). The value of the RHR depends on several factors such as the age of the person being measured [7], [8], [9], gender [7], [9], and the posture in which the measurement was taken [10]. The HR has been reported to decrease with age [8] and is higher in women than in men [7]. In terms of posture, it is about $3 \mathrm{bpm}$ higher when sitting compared to the supine position [10].

$\mathrm{HR}$ is a vital sign that can be easily and noninvasively measured without special training or equipment. Conventional non-invasive methods for cardiac activity measurement include electrocardiogram (ECG) and photoplethysmography (PPG). Both methods, although based on different concepts and measuring different phenomena, provide reliable results when used correctly [11], [12].

The ECG is a method that is based upon the perceptible electrical activity of the heart, recorded from the chest. It is considered the oldest diagnostic tool still used in medicine today. Although this method is very accurate, it has some disadvantages. The greatest of these is the need for direct contact between the electrodes and the skin. Therefore, the method is not suitable for certain groups of patients, such as 
those suffering from burns. In addition, the conductive gel used in the electrodes may cause allergic reactions. Furthermore, the electrodes must be correctly positioned, which requires a competent operator. Incorrect placement of electrodes may result in erroneous recordings. The absence of trained personnel may result in prolonged hospitalization and increased treatment costs [11], [13].

PPG is one of the plethysmographic methods. The measurement of HR with this method is based on the evaluation of volume changes caused by blood flow. It is a simple and portable technique for measuring peripheral pulse, which is indirectly related to the electrical activity of the heart through blood flow caused by muscle contraction. A simple plethysmographic device consists of a light source (usually red or near-infrared (NIR)) and a photodetector. The illuminated tissue is relatively transparent to NIR wavelengths and the level of absorption depends on hemoglobin, the oxygen transport protein that strongly absorbs NIR light [11], [12]. The variations in volume and photodetector response are related using the Beer-Lambert's law (1), which states that by illuminating tissue with an absorption coefficient $\alpha$ with a light source of intensity $\mathrm{I}_{0}$ and measuring the fraction of light received $\mathrm{I}_{\mathrm{t}}$, changes in thickness d can be assessed [14].

$$
I_{t}=I_{0} \exp (-\alpha d)
$$

During the cardiac cycle, an increase in NIR light absorbance is seen during periods of high pressure (systole), while a decrease in NIR light absorbance is seen during periods of low pressure (diastole). As systolic and diastolic phases are synchronized with HR, based on that HR can be quickly indicated [11], [12]. PPG signal can be obtained by using a pulse oximeter.

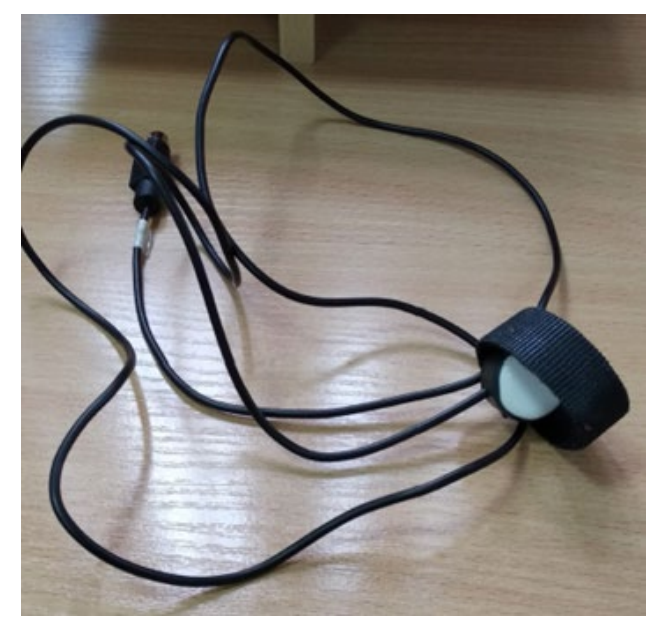

Fig.1. PTN-104 pulse plethysmograph sensor.

One of the plethysmographic sensors is the PTN-104 sensor, which is a portable and sensitive sensor composed of piezometric material, which converts pulse activity signals from blood motion to electrical signals (Fig.1.). This is a nonmagnetic accelerometer, that produces a signal from which rate and relative pressure information can be computed. The real-time integral of this signal is identical to the volume pulse signals recorded with more expensive PPGs. This sensor can be easily attached to any finger.

This sensor has been used by other investigators for diagnostic purposes, including the diagnosis of myocardial infarction [15] and dilated cardiomyopathy [16].

This study aims to assess the accuracy of the PTN-104 plethysmographic sensor in HR measurement in comparison with pulse oximetry that uses PPG.

\section{SUBJECT \& METHODS}

The accuracy of the PTN-104 sensor was tested by comparing heart rate values obtained in two different ways. The heart rate measurements to which the PTN-104 sensor results were compared were performed using a fingertip pulse oximeter. Some specifications of this sensor are shown in Table 1

Table 1. Specifications of PTN-104 sensor.

\begin{tabular}{|l|c|}
\hline \multicolumn{2}{|c|}{ PTN-104 Specifications } \\
\hline Typical pulse output [mV] & 200 \\
\hline Frequency response [Hz] & $\pm 0 / 1$ to 750 \\
\hline Connector & MINIDIN7 \\
\hline
\end{tabular}

The portable fingertip pulse oximeter has similar accuracy to the conventional hospital oximeter with a digital sensor [17]. However, there is some evidence that pulse oximeters tend to underestimate heart rate during heavy exercise $(>155$ beats/min) [18]. Therefore, the measurements were performed for heart rate at rest and after light exercise.

\subsection{Data acquisition}

The study group consisted of 12 healthy subjects. All study participants provided informed consent to be included in the study. Table 2. shows the characteristics of the study group including body mass index.

Table 2. Characteristics of the study group.

\begin{tabular}{|l|c|}
\hline \multicolumn{2}{|c|}{ Study group $\mathbf{n}=12$} \\
\hline Sex [females/males] & $6 / 6$ \\
\hline Age [years] Mean \pm SD & $20.4 \pm 1.1$ \\
\hline BMI [kg m $^{2}$ ] Mean \pm SD & $21.8 \pm 2.2$ \\
\hline
\end{tabular}

To acquire the signal, PTN-104 plethysmographic sensor in cooperation with the NI ELVIS educational platform was used. The monitored signals were converted into a numerical value by the sample LabVIEW code - iWorx Pulse.vi, which is available online [19]. This software does not allow changing any significant settings. The default value for the number of samples taken per second is 200 . The connection of the PTN-104 sensor is shown in Fig.2.

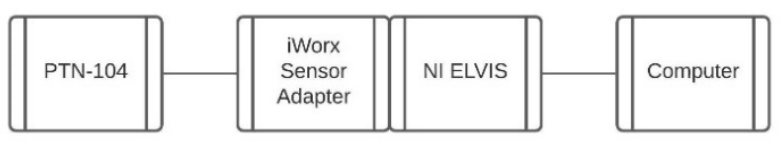

Fig.2. Connection diagram. 
The PTN-104 sensor converts changes in reflected light intensity into a signal that is processed by the mentioned VI. This raw signal is used by this VI to identify the QRS complex. The point with the highest amplitude is the $\mathrm{R}$ peak, and the interval between these two points is the RR interval. The HR is determined from the characteristic points of these waves. Each appearance of the R peak is one heartbeat. HRV, on the other hand, can be calculated from changes in RR intervals over time.

Two data sets were collected: one with resting heart rate and one with post-exercise heart rate. The PTN-104 sensor was attached to the index finger of the subjects and the subjects were in a sitting position. A fingertip pulse oximeter was attached in the same way but on the opposite hand. Resting heart rate was measured after at least 5 minutes of rest. Postexercise heart rate, on the other hand, was measured immediately after the subject performed a series of 30 squats at a specified rhythm for 1 minute (1 squat per 2 seconds).

In each dataset, a total of 36 measurements were gathered from the PTN-104 sensor and 12 measurements from the pulse oximeter. A total of 72 measurements were collected from the PTN-104 sensor and 24 from the pulse oximeter. Table 3. shows the characteristics of the acquired data in detail.

Table 3. Description of each dataset.

\begin{tabular}{|l|c|c|}
\hline & \multicolumn{2}{|c|}{ Samples } \\
\hline Sensor & $\begin{array}{c}\text { PTN-104 } \\
\text { Sensor }\end{array}$ & $\begin{array}{c}\text { Pulse } \\
\text { Oximeter }\end{array}$ \\
\hline Total & $3 /$ person & $1 /$ person \\
\hline
\end{tabular}

\subsection{Data pre-processing and statistical analysis}

To assess the level of accuracy of the PTN-104 sensor, in comparison to the PPG sensor, four levels of statistical analysis were used:

a) linear regression was used to determine the strength of the relationship between the PPG sensor and the PTN-104 sensor and whether the relationship was statistically significant. A significant correlation was identified when the p-value was less than 0.05 , while the strength of the correlation was determined by the Pearson correlation coefficient (r-value). The strength of the correlation was determined as follows: $0.9-1.0=$ strong, $0.8-0.89=$ moderately strong, $0.7-0.79=$ moderate, $0.6-0.69=$ moderately weak, $<0.59=$ weak.

b) for further evaluation of the sensor, the Bland-Altman method was used, which is a suitable way to perform a comparison between two measurement methods and also to provide quantitative measures to decide whether the new method is acceptable or not. This method became the most suitable way to determine the limits of agreement (LOA) between measurements [20]. The mean difference (MD) between the PTN-104 sensor and the PPG sensor, also known as the mean bias, was determined by first calculating the difference in the values of the individual measurements of the PTN-104 sensor and the PPG sensor (2), and then calculating the average of these differences (3).

$$
\begin{gathered}
\varepsilon=H R_{P T N}-H R_{P O} \\
M D=\frac{\sum_{i=1}^{n} \varepsilon_{i}}{n}
\end{gathered}
$$

To determine the $95 \%$ LoA, the standard deviation (SD) of the difference was first calculated and then the relationship (4) was applied.

$$
L o A=M D \pm 1.96 \cdot S D
$$

On the basis of the calculated values, plots were made.

c) the mean absolute error (MAE) between the PTN-104 sensor and the PPG sensor was computed based on (5). This represented the average difference score regardless of the direction of the difference (i.e., regardless of under- or overestimation).

$$
M A E=\frac{\sum_{i=1}^{n}\left|\varepsilon_{i}\right|}{n}
$$

d) average of relative errors $\delta$ that were calculated using the relationship (6).

$$
\delta=\frac{|\varepsilon|}{H R_{P O}} \cdot 100 \%
$$

All calculations and graphs were performed using MS Excel.

\subsection{Validity criteria}

Validity criteria for heart rate measurement were based on previous studies and consist of [21], [22]:

a correlation between the heart rate obtained from the reference device and the heart rate measured by the test device of $r=0.9$ or greater,

- a mean bias less than $3 \mathrm{bpm}$,

- a mean absolute error less than $5 \mathrm{bpm}$.

To get an accurate HR measurement, the device should meet at least the first and the second criterion.

\section{RESULTS AND DISCUSSION}

HR data from both sensors are shown in Table 4. and Table 5. For both resting and post-exercise measurements, it was noted that measurements made with the PTN-104 sensor were underestimated. The average of relative errors for the PTN-104 sensor compared to the reference method was $4.05 \%$ for HR at rest and $2.72 \%$ for post-exercise HR. 
Table 4. Comparison of values measured for resting heart.

\begin{tabular}{|c|c|c|c|c|}
\hline \multirow[b]{2}{*}{ No. } & $\begin{array}{c}\text { PTN-104 } \\
\text { Sensor }\end{array}$ & $\begin{array}{c}\text { Pulse } \\
\text { Oximeter }\end{array}$ & \multirow[b]{2}{*}{$\begin{array}{c}\mathcal{E}_{r} \\
{[\mathbf{B P M}]}\end{array}$} & \multirow[b]{2}{*}{$\begin{array}{r}\boldsymbol{\delta}_{r} \\
{[\%]}\end{array}$} \\
\hline & $\begin{array}{c}H R_{P T N} \\
{[B P M]} \\
\text { Mean } \pm \text { SD }\end{array}$ & $\begin{array}{c}H R_{P O} \\
{[B P M]}\end{array}$ & & \\
\hline 1 & $80.3 \pm 1.4$ & 87 & -6.70 & 7.70 \\
\hline 2 & $75.0 \pm 1.4$ & 79 & -4.00 & 5.06 \\
\hline 3 & $71.0 \pm 2.3$ & 78 & -7.00 & 8.97 \\
\hline 4 & $64.9 \pm 2.7$ & 65 & -0.10 & 0.15 \\
\hline 5 & $88.4 \pm 2.2$ & 90 & -1.60 & 1.78 \\
\hline 6 & $107.3 \pm 1.0$ & 107 & +0.30 & 0.28 \\
\hline 7 & $81.0 \pm 4.1$ & 89 & -8.00 & 8.99 \\
\hline 8 & $78.7 \pm 1.1$ & 81 & -2.30 & 2.84 \\
\hline 9 & $70.2 \pm 1.7$ & 77 & -6.80 & 8.83 \\
\hline 10 & $75.0 \pm 5.2$ & 77 & -2.00 & 2.60 \\
\hline 11 & $88.9 \pm 3.4$ & 89 & -0.10 & 0.11 \\
\hline 12 & $76.0 \pm 2.3$ & 77 & -1.00 & 1.30 \\
\hline
\end{tabular}

Table 5. Comparison of values measured for post-exercise heart rate.

\begin{tabular}{|c|c|c|c|c|}
\hline \multirow[b]{2}{*}{ No. } & $\begin{array}{c}\text { PTN-104 } \\
\text { Sensor }\end{array}$ & $\begin{array}{c}\text { Pulse } \\
\text { Oximeter } \\
\end{array}$ & \multirow[b]{2}{*}{$\begin{array}{c}\varepsilon_{p} \\
{[\text { BPM] }}\end{array}$} & \multirow[b]{2}{*}{$\begin{array}{r}\delta_{p} \\
{[\%]}\end{array}$} \\
\hline & \begin{tabular}{l}
\multicolumn{1}{c}{$H R_{P T N}$} \\
{$[\mathrm{BPM}]$} \\
Mean $\pm \mathrm{SD}$
\end{tabular} & $\begin{array}{c}H R_{P O} \\
{[\mathrm{BPM}]}\end{array}$ & & \\
\hline 1 & $94.0 \pm 1.1$ & 98 & -4.0 & 4.08 \\
\hline 2 & $124.0 \pm 1.4$ & 130 & -6.0 & 4.62 \\
\hline 3 & $107.0 \pm 1.4$ & 106 & +1.0 & 0.94 \\
\hline 4 & $91.5 \pm 1.4$ & 95 & -3.5 & 3.68 \\
\hline 5 & $126.0 \pm 2.9$ & 123 & +3.0 & 2.44 \\
\hline 6 & $179.0 \pm 5.6$ & 181 & -2.0 & 1.10 \\
\hline 7 & $92.2 \pm 0.8$ & 94 & -1.8 & 1.91 \\
\hline 8 & $110.0 \pm 2.1$ & 108 & +2.0 & 1.85 \\
\hline 9 & $122.4 \pm 2.5$ & 130 & -7.6 & 5.85 \\
\hline 10 & $84.9 \pm 0.5$ & 84 & +0.9 & 1.07 \\
\hline 11 & $116.0 \pm 3.4$ & 121 & -5.0 & 4.13 \\
\hline 12 & $101.0 \pm 3.1$ & 100 & +1.0 & 1.00 \\
\hline
\end{tabular}

When examining the data acquired at rest, a strong correlation between PPG and PTN-104 sensor $(r=0.942)$ (Fig.3.) was noticed. Also, when analyzing post-exercise data, a strong correlation was found between PPG and PTN104 sensors $(r=0.988)$ (Fig.4.). The correlation between the HR obtained from the reference device and the HR measured by the test device for both series of measurements is $r>0.9$. Considering this, it can be concluded that the PTN-104 sensor meets the first validation criterion. When taking into account the aggregated data, this criterion also remains fulfilled $(\mathrm{r}=$ 0.988).

Bland-Altman plots indicated that the PTN-104 sensor underestimated HR measures compared to the reference method (Fig.5. and Fig.6.). In reference to fingertip Pulse Oximeter, the PTN-104 sensor exhibited a mean bias of $3.28 \mathrm{bpm}$ for resting measurements and $-1.83 \mathrm{bpm}$ for postexercise measurements. Therefore, the second validation criterion is only met for post-exercise measurements. With increased exercise, the heart beats faster and there is more interference at the blood-sensor interface, so it is expected that the accuracy of the device will decrease as the heart accelerates [18]. Considering that, the results are surprising. Especially that other work on the validation of sensors measuring heart rate has indicated that HR measurement error tends to increase with activity intensity [23]-[25]. Increased sensor accuracy in post-exercise testing should be verified in studies on a larger group of people, where the reference device will be the gold standard method, which is the ECG.

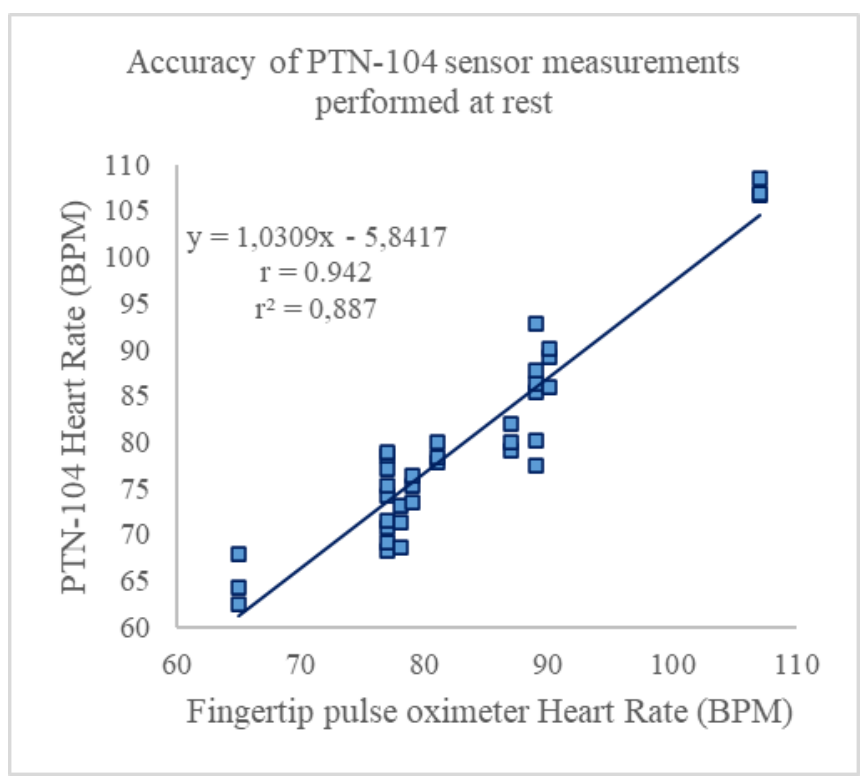

Fig.3. Linear regression line on the scatter plot relating the resting heart rate reading by the PTN-104 sensor to the pulse oximeter reading.

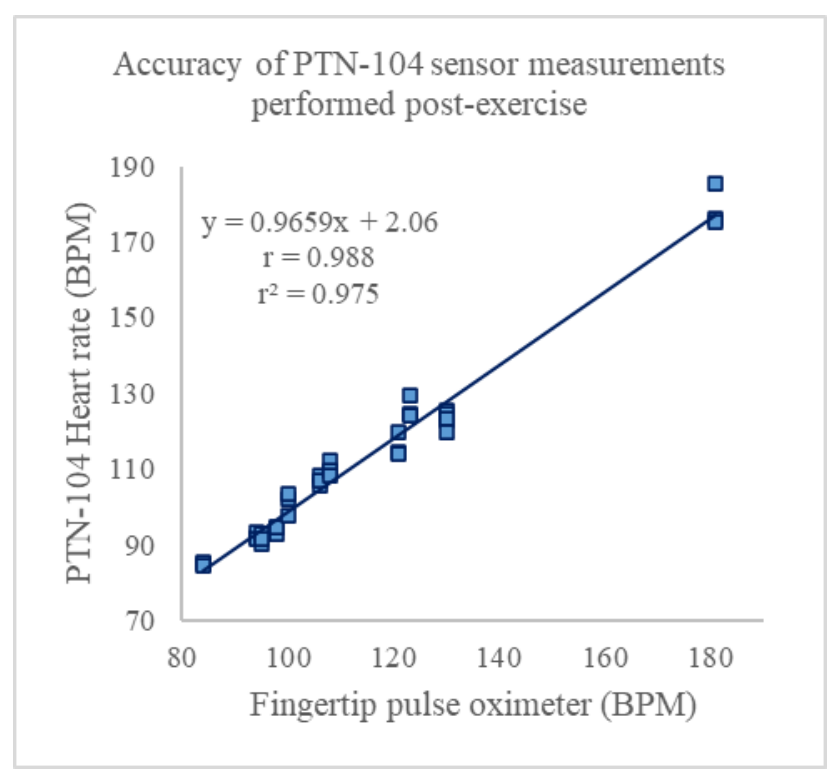

Fig.4. Linear regression line on the scatter plot relating the postexercise heart rate reading by the PTN-104 sensor to the pulse oximeter reading. 


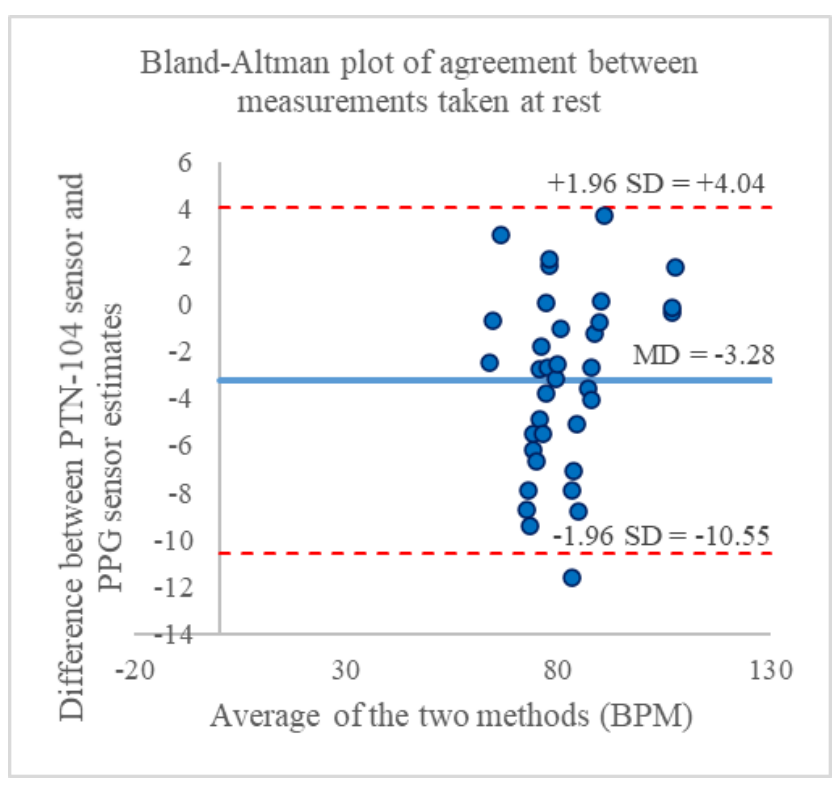

Fig.5. Bland-Altman Plot indicating mean bias scores and $95 \%$ LoA for the measurement taken at rest.

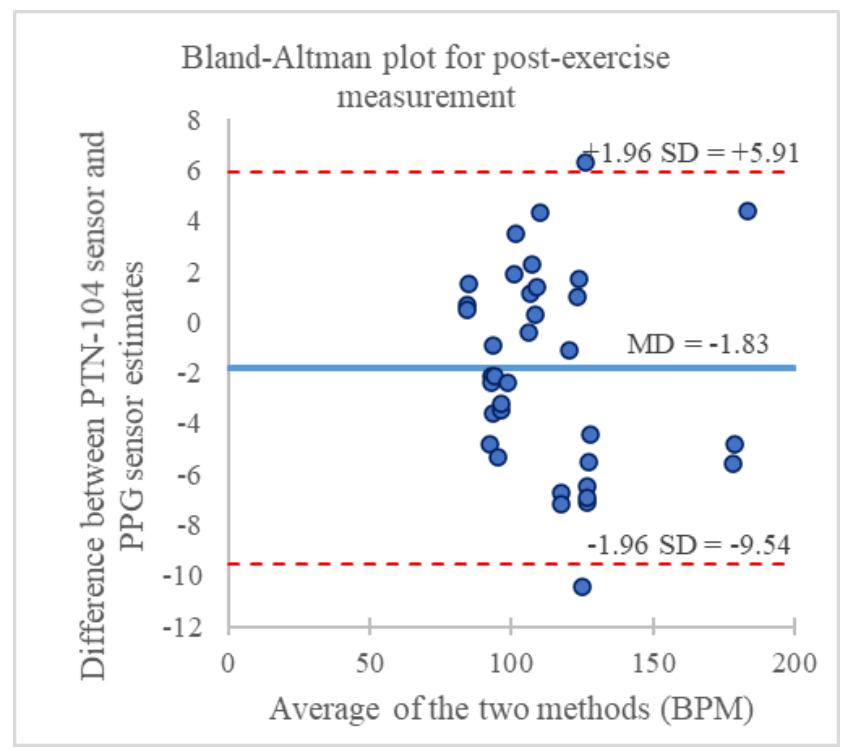

Fig.6. Bland-Altman Plot indicating mean bias scores and $95 \%$ LoA for post-exercise measurement.

Table 6. Results for mean absolute error (MAE).

\begin{tabular}{|c|c|c|}
\hline $\begin{array}{c}\text { At rest } \\
{[\mathbf{b p m}]}\end{array}$ & $\begin{array}{c}\text { Post-exercise } \\
{[\mathbf{b p m}]}\end{array}$ & $\begin{array}{c}\text { For both series } \\
{[\mathbf{b p m}]}\end{array}$ \\
\hline 3.93 & 3.55 & 3.74 \\
\hline
\end{tabular}

Taking into account the results of both measurement series, the mean bias is $-2.55 \mathrm{bpm}$. As a result, the PTN-104 sensor can be considered meeting the second criterion.

The Bland-Altman analysis reflects a tendency for the PTN104 sensor to underestimate HR. However, this tendency is smaller for higher heart rate intensities. Therefore, accuracy testing at heart rates higher than $150 \mathrm{bpm}$ should be considered.
The results for the third criterion relating to MAE are included in Table 6 . This criterion was met in all cases.

This is the first study on the accuracy of the PTN-104 sensor. Although the accuracy of this sensor has not been previously validated, it has been used for diagnostic purposes [15], [16]. This work will be helpful for future researchers who will want to use the PTN-104 sensor in their work.

The primary objective of this investigation was to assess the validity of heart rate measurements using the PTN-104 sensor. The results indicate that the sensor meets the established criteria when both series are considered as a whole. However, the sensor showed lower accuracy for measurements taken at rest, which is on the contrary to other papers. As indicated in [26], the plethysmographic sensors are less accurate compared to ECG due to wider peaks in the measured signal. Thus, the examined sensor should be compared to ECG.

According to the results, the PTN-104 sensor underestimates HR. Accurate measurements are important for accurate exercise recommendation, so the sensor studied is probably not suitable for such use. At this point, it can be stated with certainty that the investigated sensor is suitable for educational use.

\subsection{Limitations}

First of all, this is preliminary research carried out on a small study group, which included a relatively young and apparently healthy sample of participants (mean: $20.4 \pm 1.1$ years) and therefore the results may not be generalizable to the wider consumer market. Furthermore, the measurements obtained from the PTN-104 sensor were compared with those from the PPG sensor, which gives less accurate results than the ECG. Finally, it has been suggested that the accuracy of this sensor may be reduced at higher intensities, which was not considered in this study.

\section{CONCLUSIONS}

Evaluation of the PTN-104 sensor showed that it was able to measure HR at rest and after exercise, but not without errors. On average, HR measured by PTN-104 sensor was with a mean bias of $-2.55 \mathrm{bpm}$ from HR measured by PPG. Summarizing, initial results demonstrate that this sensor can be used for educational purposes. However, its applicability in clinical applications should be verified by testing it on a larger group of subjects and in comparison with the gold standard method of heart rhythm acquisition, which is the ECG.

\section{REFERENCES}

[1] Sabbah, H.N., Ilsar, I., Zaretsky, A., Rastogi, S., Wang, M., Gupta, R.C. (2011). Vagus nerve stimulation in experimental heart failure. Heart Failure Review, 16, 171-178.

[2] Böhm, M., Reil, J.-C., Deedwania, P., Kim, J.B., Borer, J.S. (2015). Resting heart rate: Risk indicator and emerging risk factor in cardiovascular disease. The American Journal of Medicine, 128 (3), 219-228. 
[3] Seravalle, G., Quarti Trevano, F., Grassi, G. (2021). Heart rate as a predictor of cardiovascular risk. Minerva Medica, 112 (1), 130-143.

[4] Lau, K., Malik, A., Foroutan, F., Buchan, T.A., Daza, J.F., Sekercioglu, N., Orchanian-Cheff, A., Alba, A.C. (2020). Resting heart rate as an important predictor of mortality \& morbidity in ambulatory patients with heart failure: A systematic review \&meta-analysis. Journal of Cardiac Failure, 27 (3), 349-363.

[5] Chen, X., Barywani, S.B., Hansson, P., Thunström, E.Ö., Rosengren, A., Ergatoudes, C., Mandalenakis, Z., Caidahl, K., Fu, M.L. (2020). Impact of changes in heart rate with age on all-cause death and cardiovascular events in 50-year-old men from the general population. Open Heart, 6 (1), e000856.

[6] Hart, J. (2015). Normal resting pulse rate ranges. Journal of Nursing Education and Practice, 5 (8), 9598.

[7] Bonnemeier H., Wiegand, U.K.H., Brandes, A., Kluge, N., Katus, H.A., Richardt, G., Potratz, J. (2003). Circadian profile of cardiac autonomic nervous modulation in healthy subjects: Differing effects of aging and gender on heart rate variability. Journal of Cardiovascular Electrophysiology, 14 (8), 791-799.

[8] Palatini, P., Benetos, A., Julius, S. (2006). Impact of increased heart rate on clinical outcomes in hypertension: Implications for antihypertensive drug therapy. Drugs, 66 (2), 133-144.

[9] Zhang, J. (2007). Effect of age and sex on heart rate variability in healthy subjects. Journal of Manipulative and Physiological Therapeutics, 30 (5), 374-379.

[10] Kristal-Boneh, E., Harari, G., Weinstein, Y., Green, M.S. (1995). Factors affecting differences in supine, sitting, and standing heart rate: The Israeli CORDIS study. Aviation Space and Environmental Medicine, 66 (8), 775-779.

[11] Kranjec, J., Beguš, S., Geršak, G., Drnovšek, J. (2014). Non-contact heart rate and heart rate variability measurements: A review. Biomedical Signal Processing and Control, 13, 102-112.

[12] De Pinho Ferreira, N., Gehin, C., Massot, B. (2021). A review of methods for non-invasive heart rate measurement on wrist. IRBM, 42 (1), 4-18.

[13] Gorgels, A.P.M. (2007). Electrocardiography. In Cardiovascular Medicine. Springer, 43-77.

[14] Nitzan, M., Babchenko, A., Khanokh, B., Taitelbaum, H. (2000). Measurement of oxygen saturation in venous blood by dynamic near IR spectroscopy. Journal of Biomedical Optics, 5 (2), 155-162.

[15] Khan, M.U., Aziz, S., Malik, A., Imtiaz, M.A. (2019). Detection of myocardial infarction using pulse plethysmograph signals. In 2019 International Conference on Frontiers of Information Technology (FIT). IEEE, 95.
[16] Khan, M.U., Aziz, S., Malik, A., Imtiaz, M.A. (2019). Detection of dilated cardiomyopathy using pulse plethysmographic signal analysis. In 2019 22nd International Multitopic Conference (INMIC). IEEE.

[17] Pupim, D., Filho, L.I., Takeshita, W.M., Iwaki, L.V. (2013). Evaluation of accuracy of portable fingertip pulse oximeter, as compared to that of a hospital oximeter with digital sensor. Indian Journal of Dental Research, 24 (5), 542-546.

[18] Iyriboz, Y., Powers, S., Morrow, J., Ayers, D., Landry, G. (1991). Accuracy of pulse oximeters in estimating heart rate at rest and during exercise. British Journal of Sports Medicine, 25 (3), 162-164.

[19] National Instruments Corp. iWorx Sensor Kits: Documentation, VIs, and Sample Labs. https://www.ni.com/gate/gb/GB_ACADIWORXSENS OR/US.

[20] Mansournia, M.A., Waters, R., Nazemipour, M., Bland, M., Altman, D.G. (2021). Bland-Altman methods for comparing methods of measurement and response to criticisms. Global Epidemiology, 3, 100045.

[21] Dolezal, B.A., Boland, D.M., Carney, J., Abrazado, M., Smith, D.L., Cooper, C.B. (2014). Validation of heart rate derived from a physiological status monitorembedded compression shirt against criterion ECG. Journal of Occupational and Environmental Hygiene, 11 (12), 833-839.

[22] Wallen, M.P., Gomersall, S.R., Keating, S.E., Wisløff, U., Coombes, J.S. (2016). Accuracy of heart rate watches: Implications for weight management. PLoS One, 11 (5), e0154420.

[23] Spierer, D.K., Rosen, Z., Litman, L.L., Fujii, K. (2015). Validation of photoplethysmography as a method to detect heart rateduring rest and exercise. Journal of Medical Engineering and Technology, 39 (5), 264-271.

[24] Jo, E., Lewis, K., Directo, D., Kim, D.J., Dolezal, B.A. (2016) Validation of biofeedback wearables for photoplethysmographic heart rate tracking. Journal of Sports Science \& Medicine, 15 (3), 540-547.

[25] Pasadyn, S.R., Soudan, M., Gillinov, M., Houghtaling, P., Phelan, D., Gillinov, N., Bittel, B., Desai, M.Y. (2019). Accuracy of commercially available heart rate monitors in athletes: A prospective study. Cardiovascular Diagnosis \& Therapy, 9 (4), 379-385.

[26] Lu, G., Yang, F. (2009). Limitations of oximetry to measure heart rate variability measures. Cardiovascular Engineering, 9, 119-125.

Received April 29, 2021

Accepted August 26, 2021 